\title{
Terminal Sliding Mode Control of Mobile Wheeled Inverted Pendulum System with Nonlinear Disturbance Observer
}

\author{
SongHyok Ri, ${ }^{1,2}$ Jian Huang, ${ }^{1}$ Yongji Wang, ${ }^{1}$ MyongHo Kim, ${ }^{2}$ and Sonchol $\mathrm{An}^{2}$ \\ ${ }^{1}$ Key Laboratory of Ministry of Education for Image Processing and Intelligent Control, School of Automation, \\ Huazhong University of Science and Technology, No. 1037, Luoyu Road, Hongshan District, Wuhan, Hubei 430074, China \\ ${ }^{2}$ Faculty of Control Science, University of Science and Technology, Unjong District, \\ Pyongyang 999093, Democratic People's Republic of Korea
}

Correspondence should be addressed to Jian Huang; huang_jan@mail.hust.edu.cn

Received 6 June 2014; Accepted 30 August 2014; Published 29 September 2014

Academic Editor: Kang Li

Copyright (C) 2014 SongHyok Ri et al. This is an open access article distributed under the Creative Commons Attribution License, which permits unrestricted use, distribution, and reproduction in any medium, provided the original work is properly cited.

A terminal sliding mode controller with nonlinear disturbance observer is investigated to control mobile wheeled inverted pendulum system. In order to eliminate the main drawback of the sliding mode control, "chattering" phenomenon, and for compensation of the model uncertainties and external disturbance, we designed a nonlinear disturbance observer of the mobile wheeled inverted pendulum system. Based on the nonlinear disturbance observer, a terminal sliding mode controller is also proposed. The stability of the closed-loop mobile wheeled inverted pendulum system is proved by Lyapunov theorem. Simulation results show that the terminal sliding mode controller with nonlinear disturbance observer can eliminate the "chattering" phenomenon, improve the control precision, and suppress the effects of external disturbance and model uncertainties effectively.

\section{Introduction}

Mobile wheeled inverted pendulum- (MWIP-) based robots are able to provide effective physical assistance to humans in various activities such as delivery and touring. Recently, many robotic systems are designed based on MWIP model, such as Segway [1], JOE [2], UW-Car [3], and PMP [4].

However, the control of the inherent unstable MWIP system is a challenge. First of all, the dynamics of MWIP system is underactuated; that is, the number of the control inputs is less than the number of the degrees of freedom to be stabilized. In addition, MWIP systems are different from either the conventional cart and pendulum systems or the conventional nonholonomic systems. Therefore, many available control design approaches are not applicable to the MWIP systems.

In the past several years many approaches have been applied in the control of MWIP, including the linear [5] or feedback linearization [6] methods, fuzzy control methods [7], neural network-based methods [8], adaptive control, and optimized model reference adaptive control [9].
The sliding mode control (SMC) approach seems an appropriate control method to deal with uncertain MWIP systems because SMC is less sensitive to model uncertainty and noise disturbances. Sankaranarayanan and Mahindrakar [10] proposed a sliding mode control algorithm to robustly stabilize a class of underactuated mechanical systems that are not linearly controllable and violate Brockett's necessary condition for smooth asymptotic stabilization of the equilibrium, with parametric uncertainties.

Park et al. [11] proposed an adaptive neural SMC method for trajectory tracking control of nonholonomic wheeled mobile robots with model uncertainties and external disturbances. Huang et al. [12] proposed a velocity control method for the MWIP based on sliding mode and a novel sliding surface.

Terminal sliding mode control (TSMC) of finite-time convergence is a variable structure control method whose formation and development are based on the introduction of a nonlinear function into sliding hyperplane. Compared to the conventional SMC, TSMC provides faster finite-time convergence and higher control precision. So far, the research 
of control methods of the terminal sliding mode control can be mainly divided into two types, that is, fractional exponent method such as $s=\dot{e}+e^{p / q}[13]$ and cubic polynomials [14]. Bayramoglu and Komurcugil $[15,16]$ proposed a nonsingular decoupled terminal sliding mode control (NDTSMC) method for a class of underactuated fourth-order nonlinear systems. This control method is relatively simple. However, these references do not involve how to choose two intermediate parameters $z_{u}$ and $\Phi_{z}$, which brings difficulty of their practical applications. Huang et al. [3], for a novel narrow vehicle based on an MWIP system and a movable seat, called UW-Car, proposed two terminal sliding mode controllers to control the velocity and braking.

Although TSMC controller is less sensitive to parameter variations and noise disturbances, its robustness is normally obtained by increasing the switch gain $k$. Note that a bigger $k$ also brings chatter to the system which is the main drawback of the SMC.

Disturbance observer might be a candidate solution for the problem. It is found that using a disturbance observer can further improve the robustness of controller. A nonlinear disturbance observer was proposed by Mohammadi et al. [17] to manage the disturbance of nonlinear system, which is applied for a 4-degree-of-freedom SCARA manipulator. Chen [18] proposed a nonlinear disturbance observer to deal with the disturbance of nonlinear system, which is applied to tracking control of pneumatic artificial muscle actuator by using DSC control method [19]. Wei et al. [20], for uncertain structural systems, proposed a new type of composite control scheme of disturbance-observer-based control and terminal sliding mode control (TSMC). Yang et al. [21], for systems with mismatched uncertainties, proposed a sliding mode control approach by using a novel sliding surface based on a disturbance observer.

However, most of the aforementioned studies rarely discussed terminal sliding mode control with disturbance observer for an underactuated system such as the MWIP.

In this paper, we proposed a terminal sliding mode controller with nonlinear disturbance observer (TSMCNDO) for the balance control of an MWIP system. Compared with the conventional sliding mode controller in [14], larger stability region, very higher control precision, and smaller chattering can be achieved by applying the TSMCNDO strategy.

The rest of this paper is organized as follows. The MWIP system formulation and nonlinear disturbance observer are discussed in Section 2. The terminal sliding mode control with nonlinear disturbance observer (TSMCNDO) and stability analysis are discussed in Section 3. Section 4 presents some MATLAB simulation results and the paper finally ends by the conclusion in Section 5 .

In the rest of this paper, $(\stackrel{\odot}{)}$ denotes a nominal value of $(\cdot)$.

\section{System Formulation}

2.1. MWIP System Dynamic Model. The MWIP system is a one-dimensional inverted pendulum that rotates about the wheels' axles. Hence, inclination and translational motion of
TABLE 1: Notations for MWIP parameters.

\begin{tabular}{ll}
\hline Parameter & Description \\
\hline$m_{b}, m_{w}$ & $\begin{array}{l}\text { Masses of the body and the wheel } \\
I_{b}, I_{w}\end{array}$ \\
$l$ & $\begin{array}{l}\text { Moments of inertia of the body and the wheel } \\
\text { Length between the wheel axle and the center of } \\
\text { gravity of the body }\end{array}$ \\
$r$ & $\begin{array}{l}\text { Radius of the wheel } \\
D_{b}\end{array}$ \\
$D_{w}$ & $\begin{array}{l}\text { Viscous resistance in the driving system } \\
u\end{array}$ \\
\hline
\end{tabular}

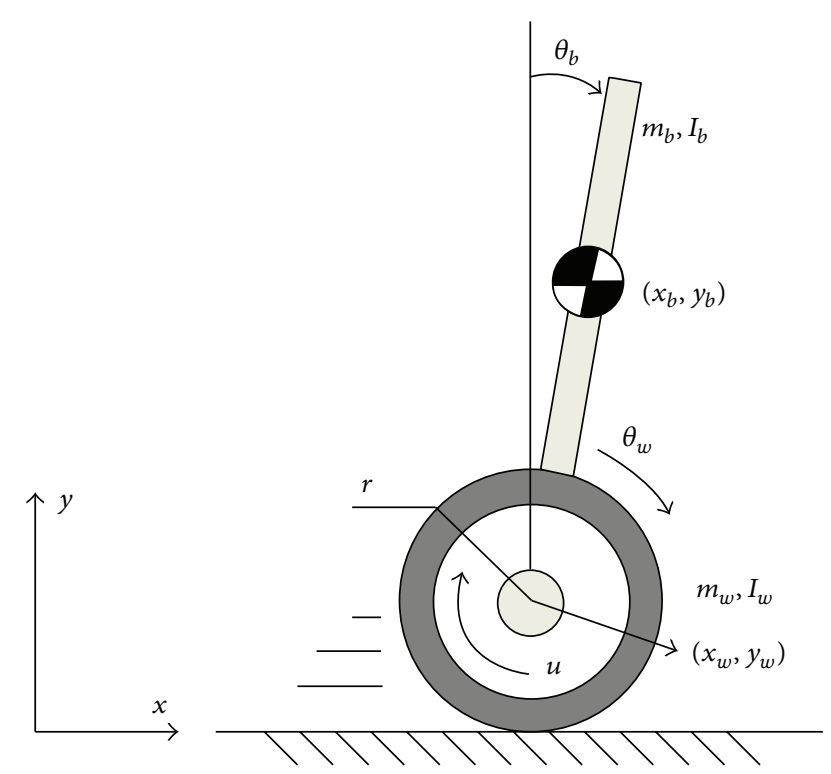

FIGURE 1: Mobile wheeled inverted pendulum (MWIP) system model.

the body determined the whole motion on a plane. Figure 1 shows the structure of an MWIP system, where $\theta_{b}$ and $\theta_{w}$ are the inclination angle of the body and the wheel's rotation angle, respectively. To describe the parameters of the MWIP system, some notations should be clarified first (see also Figure 1), which are shown in Table 1.

Lagrange's motion equation is used to analyze the dynamics of this system, which leads to a second-order underactuated model given by Huang et al. [12]. Consider

$$
\begin{aligned}
& m_{11} \ddot{\theta}_{w}+m_{12} \cos \left(\theta_{b}\right) \ddot{\theta}_{b} \\
& =u-\left(D_{w}+D_{b}\right) \dot{\theta}_{w}+D_{b} \dot{\theta}_{b}+m_{12} \dot{\theta}_{b}^{2} \sin \left(\theta_{b}\right), \\
& m_{12} \cos \left(\theta_{b}\right) \ddot{\theta}_{w}+m_{22} \ddot{\theta}_{b} \\
& =-u-D_{b}\left(\dot{\theta}_{b}-\dot{\theta}_{w}\right)+G_{b} \sin \left(\theta_{b}\right),
\end{aligned}
$$

where parameters $m_{11}, m_{12}, m_{22}$, and $G_{b}$ satisfy

$$
\begin{aligned}
& m_{11}=\left(m_{b}+m_{w}\right) r^{2}+I_{w}, \\
& m_{12}=m_{b} l r,
\end{aligned}
$$




$$
\begin{aligned}
m_{22} & =m_{b} l^{2}+I_{b}, \\
G_{b} & =m_{b} g l .
\end{aligned}
$$

Add the first equation of (1) to the second one and consider external disturbance; we have

$$
\begin{aligned}
& m_{11} \ddot{\theta}_{w}+m_{12} \cos \left(\theta_{b}\right) \ddot{\theta}_{b} \\
& \quad=u-\left(D_{w}+D_{b}\right) \dot{\theta}_{w}+D_{b} \dot{\theta}_{b}+m_{12} \dot{\theta}_{b}^{2} \sin \left(\theta_{b}\right)+\tau_{\mathrm{ex}}, \\
& \left(m_{11}+m_{12} \cos \left(\theta_{b}\right)\right) \ddot{\theta}_{w}+\left(m_{22}+m_{12} \cos \left(\theta_{b}\right)\right) \ddot{\theta}_{b} \\
& \quad=-D_{w} \dot{\theta}_{w}+m_{12} \dot{\theta}_{b}^{2} \sin \left(\theta_{b}\right)+G_{b} \sin \left(\theta_{b}\right),
\end{aligned}
$$

where $\tau_{\text {ex }}$ are used to denote external disturbance.

2.2. Nonlinear Disturbance Observer Design. In order to improve the robustness and control precision of the MWIP system, it is necessary to design a nonlinear disturbance observer estimating model uncertainties and external disturbance. This subsection illustrates the design procedure of a nonlinear disturbance observer in the MWIP system.

For the nonlinear underactuated system with disturbances, in order to simplify the denotation, we can rewrite (3) as

$$
M(q) \ddot{q}+N(q, \dot{q})+F(\dot{q})=\tau+\tau_{\text {ext }},
$$

where

$$
\begin{gathered}
q=\left[\begin{array}{ll}
q_{1} & q_{2}
\end{array}\right]^{T}=\left[\begin{array}{ll}
\theta_{w} & \theta_{b}
\end{array}\right]^{T}, \\
M(q)=\left[\begin{array}{cc}
m_{11} & m_{12} \cos \left(q_{2}\right) \\
m_{11}+m_{12} \cos \left(q_{2}\right) & m_{22}+m_{12} \cos \left(q_{2}\right)
\end{array}\right], \\
N(q, \dot{q})=\left[\begin{array}{c}
-m_{12} \dot{q}_{2}^{2} \sin \left(q_{2}\right) \\
-G_{b} \sin \left(q_{2}\right)-m_{12} \dot{q}_{2}^{2} \sin \left(q_{2}\right)
\end{array}\right], \\
F(\dot{q})=\left[\begin{array}{c}
\left(D_{w}+D_{b}\right) \dot{q}_{1}-D_{b} \dot{q}_{2} \\
D_{w} \dot{q}_{1}
\end{array}\right], \\
\tau=\left[\begin{array}{c}
u \\
0
\end{array}\right], \quad \tau_{\mathrm{ext}}=\left[\begin{array}{c}
\tau_{\mathrm{ex}} \\
0
\end{array}\right] .
\end{gathered}
$$

Then, we can get

$$
\begin{gathered}
M(q)=\widehat{M}(q)+\Delta M(q), \\
N(q, \dot{q})=\widehat{N}(q, \dot{q})+\Delta N(q, \dot{q}) .
\end{gathered}
$$

The lumped disturbance vector $\tau_{d}$ is defined as

$$
\tau_{d}=\left[\begin{array}{ll}
\tau_{d 1} & \tau_{d 2}
\end{array}\right]^{T}=\tau_{\mathrm{ext}}-\Delta M(q) \ddot{q}-\Delta N(q, \dot{q})-F(\dot{q}) .
$$

Therefore, the effect of all modelling uncertainties and external disturbance is lumped into a single disturbance vector $\tau_{d}$. From (4), it is seen that

$$
\widehat{M}(q) \ddot{q}+\widehat{N}(q, \dot{q})=\tau+\tau_{d} .
$$

To estimate the lumped disturbance $\tau_{d}$, the nonlinear disturbance observer is designed as follows:

$$
\dot{\hat{\tau}}_{d}=-L \widehat{\tau}_{d}+L(\widehat{M}(q) \ddot{q}+\widehat{N}(q, \dot{q})-\tau) .
$$

Define $\widetilde{\tau}_{d}=\tau_{d}-\widehat{\tau}_{d}$ as the disturbance tracking error and using (9), it is observed that

$$
\dot{\hat{\tau}}_{d}=L \tilde{\tau}_{d}
$$

or, equivalently,

$$
\dot{\tilde{\tau}}_{d}=\dot{\tau}_{d}-L \widetilde{\tau}_{d} .
$$

In general, there is no prior information about the derivative of the disturbance $\tau_{d}$. When the disturbance varies slowly relative to the observer dynamics, it is reasonable to suppose that $\dot{\tau}_{d}=0$. Then, we get

$$
\dot{\tilde{\tau}}_{d}=-\dot{\hat{\tau}}_{d}=-L \widetilde{\tau}_{d} .
$$

Let us define an auxiliary variable $z=\left[\begin{array}{ll}z_{1} & z_{2}\end{array}\right]^{T}=\widehat{\tau}_{d}-p(q, \dot{q})$, where $(d / d t) p(q, \dot{q})=L(q, \dot{q}) \widehat{M}(q) \ddot{q}$. Substitute it into (9); the observer can be designed as

$$
\begin{gathered}
\dot{z}=L(q, \dot{q})[\widehat{N}(q, \dot{q})-\tau-p(q, \dot{q})-z], \\
\widehat{\tau}_{d}=z+p(q, \dot{q}),
\end{gathered}
$$

where observer gain matrix $L(q, \dot{q})$ and vector $p(q, \dot{q})$ satisfy

$$
\begin{gathered}
L(q)=X \widehat{M}^{-1}(q), \\
p(\dot{q})=X \dot{q} .
\end{gathered}
$$

$X$ is a constant invertible matrix; that is,

$$
X=\left[\begin{array}{ll}
c_{1} & c_{2} \\
c_{3} & c_{4}
\end{array}\right], \quad c_{i} \geq 0, \quad i=1,2,3,4 .
$$

Substituting (14) and (15) into (13) and using (4) we have

$\dot{z}$

$$
\begin{aligned}
& =\left[\begin{array}{l}
\dot{z}_{1} \\
\dot{z}_{2}
\end{array}\right] \\
& =A^{-1}\left[\begin{array}{ll}
c_{1} & c_{2} \\
c_{3} & c_{4}
\end{array}\right]\left[\begin{array}{cc}
\widehat{m}_{22}+\widehat{m}_{12} \cos \left(\theta_{b}\right) & -\widehat{m}_{12} \cos \left(\theta_{b}\right) \\
-\widehat{m}_{11}-\widehat{m}_{12} \cos \left(\theta_{b}\right) & \widehat{m}_{11}
\end{array}\right] \\
& \cdot\left\{\left[\begin{array}{c}
-\widehat{m}_{12} \dot{\theta}_{b}^{2} \sin \left(\theta_{b}\right) \\
-\widehat{G}_{b} \sin \left(\theta_{b}\right)-\widehat{m}_{12} \dot{\theta}_{b}^{2} \sin \left(\theta_{b}\right)
\end{array}\right]\right. \\
& \left.-\left[\begin{array}{l}
u \\
0
\end{array}\right]-\left[\begin{array}{ll}
c_{1} & c_{2} \\
c_{3} & c_{4}
\end{array}\right]\left[\begin{array}{l}
\dot{\theta}_{w} \\
\dot{\theta}_{b}
\end{array}\right]-\left[\begin{array}{l}
z_{1} \\
z_{2}
\end{array}\right]\right\} \\
& =A^{-1}\left[\begin{array}{ll}
c_{1} D_{1}-c_{2} D_{2} & c_{2} \widehat{m}_{11}-c_{1} \widehat{m}_{12} \cos \left(\theta_{b}\right) \\
c_{3} D_{1}-c_{4} D_{2} & c_{4} \widehat{m}_{11}-c_{3} \widehat{m}_{12} \cos \left(\theta_{b}\right)
\end{array}\right] \\
& \cdot\left[\begin{array}{c}
-\widehat{m}_{12} \dot{\theta}_{b}^{2} \sin \left(\theta_{b}\right)-u-c_{1} \dot{\theta}_{w}-c_{2} \dot{\theta}_{b}-z_{1} \\
-\widehat{G}_{b} \sin \left(\theta_{b}\right)-\widehat{m}_{12} \dot{\theta}_{b}^{2} \sin \left(\theta_{b}\right)-c_{3} \dot{\theta}_{w}-c_{4} \dot{\theta}_{b}-z_{2}
\end{array}\right], \\
& \widehat{\tau}_{d}=\left[\begin{array}{l}
\widehat{\tau}_{d 1} \\
\widehat{\tau}_{d 2}
\end{array}\right]=\left[\begin{array}{l}
z_{1} \\
z_{2}
\end{array}\right]+\left[\begin{array}{ll}
c_{1} & c_{2} \\
c_{3} & c_{4}
\end{array}\right]\left[\begin{array}{l}
\dot{\theta}_{w} \\
\dot{\theta}_{b}
\end{array}\right],
\end{aligned}
$$


where

$$
\begin{gathered}
D_{1}=\widehat{m}_{22}+\widehat{m}_{12} \cos \left(\theta_{b}\right), \\
D_{2}=\widehat{m}_{11}+\widehat{m}_{12} \cos \left(\theta_{b}\right), \\
A=\operatorname{det}(\widehat{M}(q))=\widehat{m}_{11} \widehat{m}_{22}-\left(\widehat{m}_{12} \cos \left(\theta_{b}\right)\right)^{2}>0 .
\end{gathered}
$$

Therefore, the disturbance observer can be designed as follows:

$$
\begin{aligned}
& \dot{z}_{1} \\
& =A^{-1}\left[\left(c_{2} D_{2}-c_{1} D_{1}\right)\right. \\
& \cdot\left(\widehat{m}_{12} \dot{\theta}_{b}^{2} \sin \left(\theta_{b}\right)+u+c_{1} \dot{\theta}_{w}+c_{2} \dot{\theta}_{b}+z_{1}\right) \\
& -\left(c_{2} \widehat{m}_{11}-c_{1} \widehat{m}_{12} \cos \left(\theta_{b}\right)\right) \\
& \left.\times\left(\widehat{G}_{b} \sin \left(\theta_{b}\right)+\widehat{m}_{12} \dot{\theta}_{b}^{2} \sin \left(\theta_{b}\right)+c_{3} \dot{\theta}_{w}+c_{4} \dot{\theta}_{b}+z_{2}\right)\right], \\
& \dot{z}_{2} \\
& =A^{-1}\left[\left(c_{4} D_{2}-c_{3} D_{1}\right)\right. \\
& \cdot\left(\widehat{m}_{12} \dot{\theta}_{b}^{2} \sin \left(\theta_{b}\right)+u+c_{1} \dot{\theta}_{w}+c_{2} \dot{\theta}_{b}+z_{1}\right) \\
& \text { - }\left(c_{4} \widehat{m}_{11}-c_{3} \widehat{m}_{12} \cos \left(\theta_{b}\right)\right) \\
& \left.\times\left(\widehat{G}_{b} \sin \left(\theta_{b}\right)+\widehat{m}_{12} \dot{\theta}_{b}^{2} \sin \left(\theta_{b}\right)+c_{3} \dot{\theta}_{w}+c_{4} \dot{\theta}_{b}+z_{2}\right)\right], \\
& \widehat{\tau}_{d 1}=z_{1}+c_{1} \dot{\theta}_{w}+c_{2} \dot{\theta}_{b} \\
& \widehat{\tau}_{d 2}=z_{2}+c_{3} \dot{\theta}_{w}+c_{4} \dot{\theta}_{b} \text {. }
\end{aligned}
$$

\section{Controller Design}

The MWIP system model (3) can be rewritten as

$$
\begin{gathered}
\widehat{m}_{11} \ddot{\theta}_{w}+\widehat{m}_{12} \cos \left(\theta_{b}\right) \ddot{\theta}_{b}=u+\widehat{m}_{12} \dot{\theta}_{b}^{2} \sin \left(\theta_{b}\right)+\tau_{d 1}, \\
\left(\widehat{m}_{11}+\widehat{m}_{12} \cos \left(\theta_{b}\right)\right) \ddot{\theta}_{w}+\left(\widehat{m}_{22}+\widehat{m}_{12} \cos \left(\theta_{b}\right)\right) \ddot{\theta}_{b} \\
=\widehat{m}_{12} \dot{\theta}_{b}^{2} \sin \left(\theta_{b}\right)+\widehat{G}_{b} \sin \left(\theta_{b}\right)+\tau_{d 2} .
\end{gathered}
$$

From the two equations of (19), we have

$$
\begin{aligned}
\ddot{\theta}_{b}= & A^{-1}\left[\widehat{m}_{11} \widehat{G}_{b} \sin \left(\theta_{b}\right)-\widehat{m}_{12}^{2} \dot{\theta}_{b}^{2} \sin \left(\theta_{b}\right) \cos \left(\theta_{b}\right)\right] \\
& -A^{-1}\left(\widehat{m}_{11}+\widehat{m}_{12} \cos \left(\theta_{b}\right)\right) u \\
& +A^{-1}\left[\widehat{m}_{11} \tau_{d 2}-\left(\widehat{m}_{11}+\widehat{m}_{12} \cos \left(\theta_{b}\right)\right) \tau_{d 1}\right] .
\end{aligned}
$$

According to the TSMC method proposed in [14], the sliding surface is defined as follows:

$$
\mathbf{s}(t)=\dot{\mathbf{e}}(t)+\operatorname{Ce}(t)-\mathbf{w}(t),
$$

where $\mathbf{e}(t)=\mathbf{x}(t)-\mathbf{x}_{\mathbf{d}}(t)$, and $\mathbf{x}_{\mathbf{d}}(t)$ is the reference value. And,

$$
\begin{gathered}
\mathbf{C}=\operatorname{diag}\left(c_{1}, c_{2}, \ldots, c_{m}\right), \quad c_{i}>0, \\
\mathbf{w}(t)=\dot{\mathbf{v}}(t)+\mathbf{C v}(t) .
\end{gathered}
$$

The TSMC method is applied to the subsystem (20). The desired inclination angle $\theta_{b}^{*}$ and its rate of change $\dot{\theta}_{b}^{*}$ are expected to be zero. Let us define the following sliding surfaces:

$$
S(t)=\dot{\theta}_{b}(t)+c \theta_{b}(t)-\dot{v}(t)-c v(t),
$$

where $c$ is a positive constant. The augmenting function $v(t)$ is designed as cubic polynomials that guarantee Assumption 1 in [14] holds.

Assumption 1. The tracking errors of the nonlinear disturbance observer are bounded and satisfy

$$
\left|\tilde{\tau}_{d 1}\right| \leq d_{1}, \quad\left|\tilde{\tau}_{d 2}\right| \leq d_{2},
$$

where $d_{1}$ and $d_{2}$ are known bounds.

Theorem 2. The sliding surfaces (23) will be achieved while the inclination angle $\theta_{b}$ converges to zero in finite time if the following control law is applied to the subsystem (20):

$$
\begin{aligned}
u=\left(\widehat{m}_{11}\right. & \left.+\widehat{m}_{12} \cos \left(\theta_{b}\right)\right)^{-1} \\
\times[ & \widehat{m}_{11} \widehat{G}_{b} \sin \left(\theta_{b}\right)-\widehat{m}_{12}^{2} \dot{\theta}_{b}^{2} \sin \left(\theta_{b}\right) \cos \left(\theta_{b}\right)+\widehat{m}_{11} \widehat{\tau}_{d 2} \\
& \quad-\left(\widehat{m}_{11}+\widehat{m}_{12} \cos \left(\theta_{b}\right)\right) \widehat{\tau}_{d 1} \\
& \left.+A\left(c \dot{\theta}_{b}-\ddot{v}-c \dot{v}\right)+k \operatorname{sgn}(S)\right],
\end{aligned}
$$

where

$$
k=\gamma+\left[\widehat{m}_{11} d_{2}+\left(\widehat{m}_{11}+\widehat{m}_{12} \cos \left(\theta_{b}\right)\right) d_{1}\right], \quad \gamma>0 .
$$

Proof. Choose the following Lyapunov function candidate:

$$
V=\frac{1}{2} S^{2}
$$

Differentiating (27) along the controlled system (20) yields

$$
\begin{aligned}
\dot{V}=S \dot{S} & =S\left[\ddot{\theta}_{b}+c \dot{\theta}_{b}-\ddot{v}-c \dot{v}\right] \\
=S[ & A^{-1}\left(\widehat{m}_{11} \widehat{G}_{b} \sin \left(\theta_{b}\right)-\widehat{m}_{12}^{2} \dot{\theta}_{b}^{2} \sin \left(\theta_{b}\right) \cos \left(\theta_{b}\right)\right) \\
& -A^{-1}\left(\widehat{m}_{11}+\widehat{m}_{12} \cos \left(\theta_{b}\right)\right) u \\
& +A^{-1}\left[\widehat{m}_{11} \tau_{d 2}-\left(\widehat{m}_{11}+\widehat{m}_{12} \cos \left(\theta_{b}\right)\right) \tau_{d 1}\right] \\
& \left.+c \dot{\theta}_{b}-\ddot{v}-c \dot{v}\right] .
\end{aligned}
$$

Substituting (25) into (28)

$\dot{V}$

$$
\begin{aligned}
=S\{ & -A^{-1} k \operatorname{sgn}(S) \\
& \left.+A^{-1}\left[\widehat{m}_{11} \widetilde{\tau}_{d 2}-\left(\widehat{m}_{11}+\widehat{m}_{12} \cos \left(\theta_{b}\right)\right) \widetilde{\tau}_{d 1}\right]\right\} \\
=- & A^{-1} S\left\{k \operatorname{sgn}(S)-\left[\widehat{m}_{11} \widetilde{\tau}_{d 2}-\left(\widehat{m}_{11}+\widehat{m}_{12} \cos \left(\theta_{b}\right)\right) \widetilde{\tau}_{d 1}\right]\right\} \\
= & -k A^{-1}|S|+A^{-1} S\left[\widehat{m}_{11} \widetilde{\tau}_{d 2}-\left(\widehat{m}_{11}+\widehat{m}_{12} \cos \left(\theta_{b}\right)\right) \widetilde{\tau}_{d 1}\right] \\
\leq & -k A^{-1}|S|+A^{-1}|S|\left|\widehat{m}_{11} \widetilde{\tau}_{d 2}-\left(\widehat{m}_{11}+\widehat{m}_{12} \cos \left(\theta_{b}\right)\right) \widetilde{\tau}_{d 1}\right|
\end{aligned}
$$


TABle 2: Physical parameters of MWIP system.

\begin{tabular}{lccc}
\hline Parameter & Value & Parameter & Value \\
\hline$m_{w}$ & $29.0(\mathrm{Kg})$ & $I_{w}$ & $0.6\left(\mathrm{Kg} \cdot \mathrm{m}^{2}\right)$ \\
$r$ & $0.254(\mathrm{~m})$ & $\widehat{m}_{b}$ & $210.6(\mathrm{Kg})$ \\
$\widehat{I}_{b}$ & $55.0\left(\mathrm{Kg} \cdot \mathrm{m}^{2}\right)$ & $\widehat{l}$ & $0.267(\mathrm{~m})$ \\
$m_{b}$ & $310.6(\mathrm{Kg})$ & $I_{b}$ & $65.0\left(\mathrm{Kg} \cdot \mathrm{m}^{2}\right)$ \\
$l$ & $0.317(\mathrm{~m})$ & $D_{b}$ & $0.1(\mathrm{~N} \cdot \mathrm{s} / \mathrm{m})$ \\
$D_{w}$ & $4.0(\mathrm{~N} \cdot \mathrm{s} / \mathrm{m})$ & & \\
\hline
\end{tabular}

$$
\begin{aligned}
& \leq-k A^{-1}|S|+A^{-1}|S|\left[\left|\widehat{m}_{11} \tilde{\tau}_{d 2}\right|+\left|\left(\widehat{m}_{11}+\widehat{m}_{12} \cos \left(\theta_{b}\right)\right) \tilde{\tau}_{d 1}\right|\right] \\
& \leq-k A^{-1}|S|+A^{-1}|S|\left[\widehat{m}_{11} d_{2}+\left(\widehat{m}_{11}+\widehat{m}_{12} \cos \left(\theta_{b}\right)\right) d_{1}\right] \\
& \leq-A^{-1}|S|\left(k-\left[\widehat{m}_{11} d_{2}+\left(\widehat{m}_{11}+\widehat{m}_{12} \cos \left(\theta_{b}\right)\right) d_{1}\right]\right) \\
& =-\gamma A^{-1}|S|<0 .
\end{aligned}
$$

Therefore, $V$ is a positive-definite function and $\dot{V}$ is a negative-definite function. From Remark 1 in [14], it is easily known that $S(0)=0$, and it implies that $V(0)=0$. Thus, it implies that $V(t) \equiv 0$ and $S(t) \equiv 0$. This completes the proof.

Theorem 3. For the internal dynamic model of the MWIP system (the second equation of (3)), the proposed TSMCNDO controller (25) guarantees that the angular velocity $\dot{\theta}_{w}$ can converge to zero.

Proof. Similar to Proposition 2 in [3], the second equation of (3) can be rewritten as

$$
Q(t) \ddot{\theta}_{w}(t)+D_{w} \dot{\theta}_{w}(t)=P(t),
$$

where $Q(t)=m_{11}+m_{12} \cos \left(\theta_{b}\right)>0$ and $D_{w}>0$. Therefore, we can know, the solution of (30) is asymptotically stable. From Theorem 2, it follows that $\theta_{b}$ and $\dot{\theta}_{b}$ converge to zero, which makes $P(t)$ finally converge to zero. This results in the final convergence of $\dot{\theta}_{w}$.

\section{Simulation Study}

In order to verify the performance of the proposed controller, we present some simulations in this section. In the simulations, the nominal values of system parameters come from a real MWIP-based vehicle. All the parameters are given in Table 2.

The external disturbance is assumed as

$$
\tau_{\mathrm{ex}}=100 \sin \left(2 t+\frac{\pi}{2}\right)(\mathrm{N} \cdot \mathrm{m}) .
$$

The augmenting function $v(t)$ is designed as a cubic polynomial:

$$
v(t)= \begin{cases}a_{0}+a_{1} t+a_{2} t^{2}+a_{3} t^{3}, & \text { if } 0 \leq t \leq T_{f}, \\ 0, & \text { if } t>T_{f},\end{cases}
$$

TABLE 3: Control parameters of TSMC and TSMCNDO strategies.

\begin{tabular}{lc}
\hline Controllers & Parameters \\
\hline TSMC & $F=0.7 \widehat{F}, C=3, \Gamma=3, D=0.3, \phi_{\text {TSMC }}=0.05$ \\
TSMCNDO & $\gamma=3, c=3, d_{1}=1, d_{2}=1, c_{1}=c_{4}=2000$, \\
& $c_{2}=c_{3}=0, \phi_{\text {TSMCNDO }}=0.05$ \\
\hline
\end{tabular}

where

$$
\begin{gathered}
a_{0}=\theta_{b}(0), \quad a_{1}=\dot{\theta}_{b}(0), \\
a_{2}=-3\left(\frac{\theta_{b}(0)}{T_{f}^{2}}\right)-2\left(\frac{\dot{\theta}_{b}(0)}{T_{f}}\right), \\
a_{3}=2\left(\frac{\theta_{b}(0)}{T_{f}^{3}}\right)+\left(\frac{\dot{\theta}_{b}(0)}{T_{f}^{2}}\right) .
\end{gathered}
$$

For the sliding surface, $T_{f}=1$ were used.

Suppose the initial conditions are given by $\theta_{b}=$ $-10 / 180 \pi, \dot{\theta}_{b}=0, \theta_{w}=0$, and $\dot{\theta}_{w}=0$.

We consider the balance control of MWIP systems by using the conventional linear quadratic regulator (LQR), the TSMC in [14], and the TSMCNDO proposed in this paper. When using the LQR controller, an approximately linearized dynamic model was established by choosing state as $\mathbf{x}=\left[x_{1}, x_{2}, x_{3}\right]^{T}=\left[\dot{\theta}_{w}, \theta_{b}, \dot{\theta}_{b}\right]^{T}$. The calculated state feedback gain matrix of LQR controller is $\mathbf{K}=$ [-4.3141, -962.2, -255.2].

In order to avoid chattering associated with the terminal sliding mode control law, we have approximated the discontinuous sign function $\operatorname{sgn}(S)$ with continuous saturation function sat $(S)$ defined as

$$
\operatorname{sat}(S)= \begin{cases}\operatorname{sgn}(S) & \text { if }|S|>\phi, \\ \frac{S}{\phi} & \text { if }|S| \leq \phi, \\ \phi & >0,\end{cases}
$$

where $\phi$ is boundary layer. For applying the two control strategies to the subsystem (20), the determined parameters of all controllers are listed in Table 3.

The balance control simulation results of the MWIP system with uncertainties and disturbances using the LQR, the TSMC given by [14], and the TSMCNDO proposed in this paper are shown in Figures 2, 3, 4, and 5.

Figure 6 shows the inclination angle tracking errors of the MWIP system considering uncertainties and disturbances using the two control strategies.

From Figures 2-6 the following turn out.

(1) The control performance of LQR controller seems worst because it is not designed to deal with the model uncertainties and external disturbance.

(2) Even if there are model uncertainties and external disturbance, the inclination angle, angular velocity, and wheel rotation velocity of the MWIP system will finally converge when either TSMC or TSMCNDO controller is employed. 


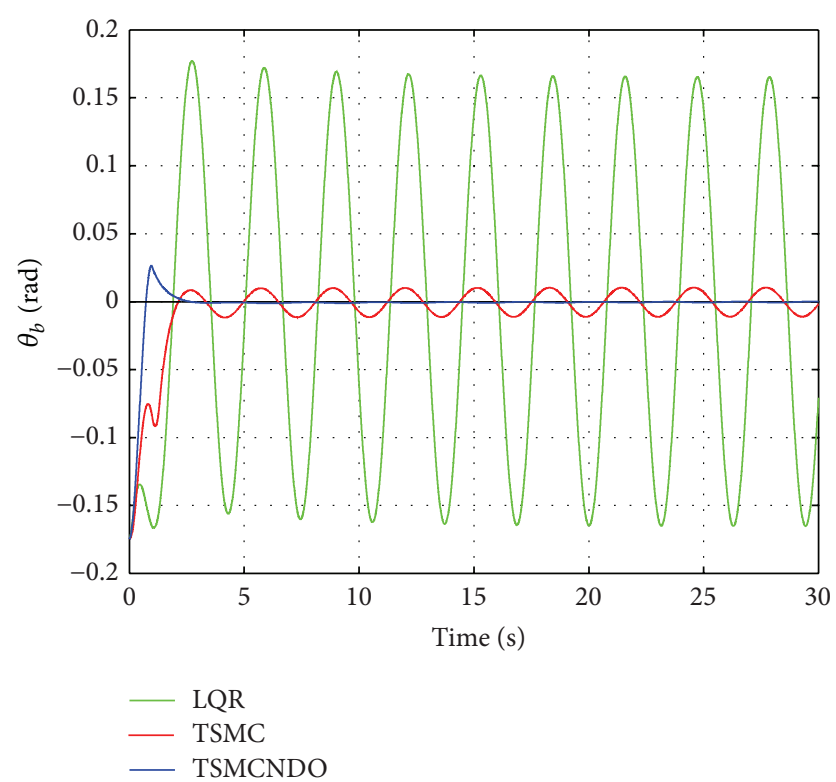

FIGURE 2: Inclination angles of the MWIP system by employing LQR, TSMC, and TSMCNDO control strategies $\left(\theta_{b}(0)=-10^{\circ}\right.$, $\phi_{\text {TSMC }}=\phi_{\text {TSMCNDO }}=0.05$ ).

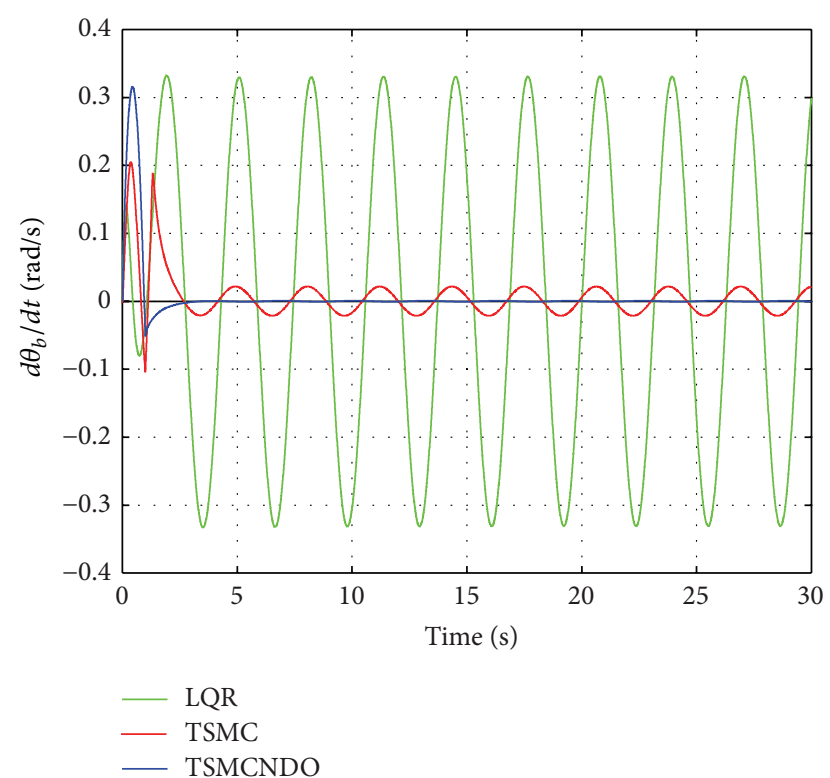

FIGURE 3: Inclination angle velocities of the MWIP system by employing LQR, TSMC, and TSMCNDO control strategies $\left(\theta_{b}(0)=\right.$ $-10^{\circ}, \phi_{\text {TSMC }}=\phi_{\text {TSMCNDO }}=0.05$ ).

(3) The control performance of the MWIP system by using TSMCNDO control strategy is better than the one by using TSMC control strategy. The effect of external disturbance on the MWIP system is significantly reduced by using TSMCNDO control strategy while it still remains when using a conventional TSMC controller.

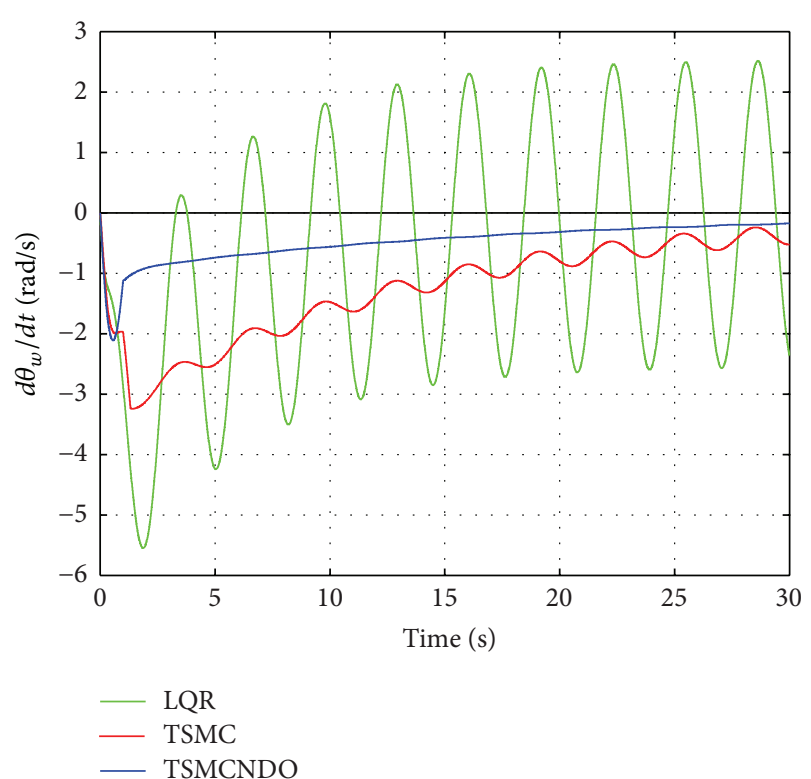

FIGURE 4: Wheel rotation velocities of the MWIP system by employing LQR, TSMC, and TSMCNDO control strategies $\left(\theta_{b}(0)=\right.$ $\left.-10^{\circ}, \phi_{\text {TSMC }}=\phi_{\text {TSMCNDO }}=0.05\right)$.

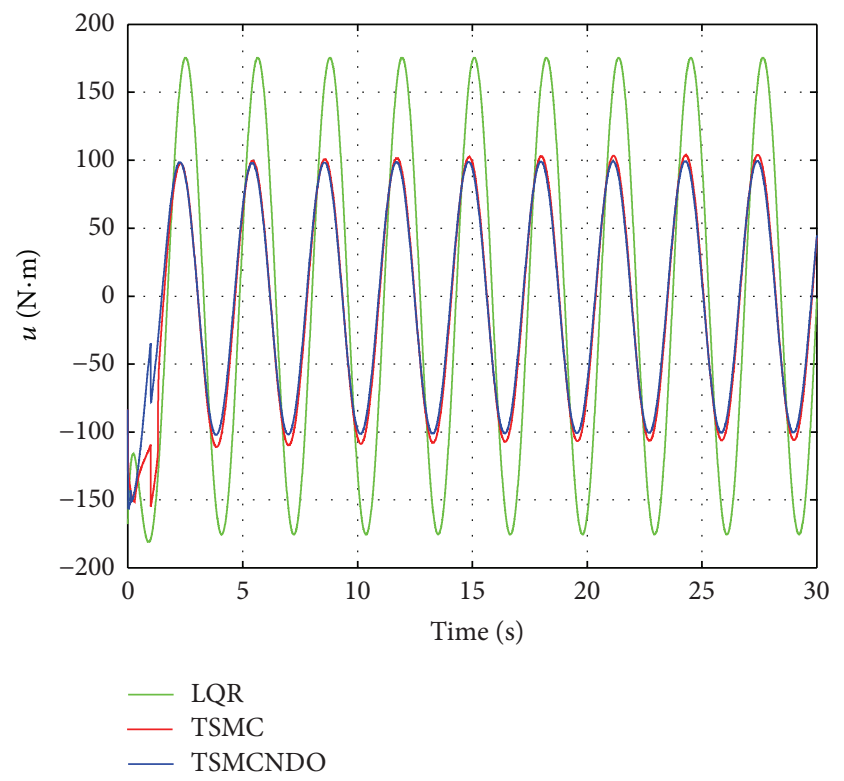

FIGURE 5: Control inputs of the MWIP system by employing LQR, TSMC, and TSMCNDO control strategies $\left(\theta_{b}(0)=-10^{\circ}, \phi_{\mathrm{TSMC}}=\right.$ $\phi_{\text {TSMCNDO }}=0.05$ ).

In the case of TSMC strategy, the control precision of the system is mainly related to parameter $\phi_{\mathrm{TSMC}}$. In order to improve the control precision of the MWIP system, usually smaller value of the parameter $\phi_{\text {TSMC }}$ should be chosen. However, the chattering will increase as the value of $\phi_{\text {TSMC }}$ decreases. On the other hand, when using the TSMCNDO strategy, a satisfactory control performance can be easily achieved even if the value of parameter $\phi_{\text {TSMCNDO }}$ is relatively 


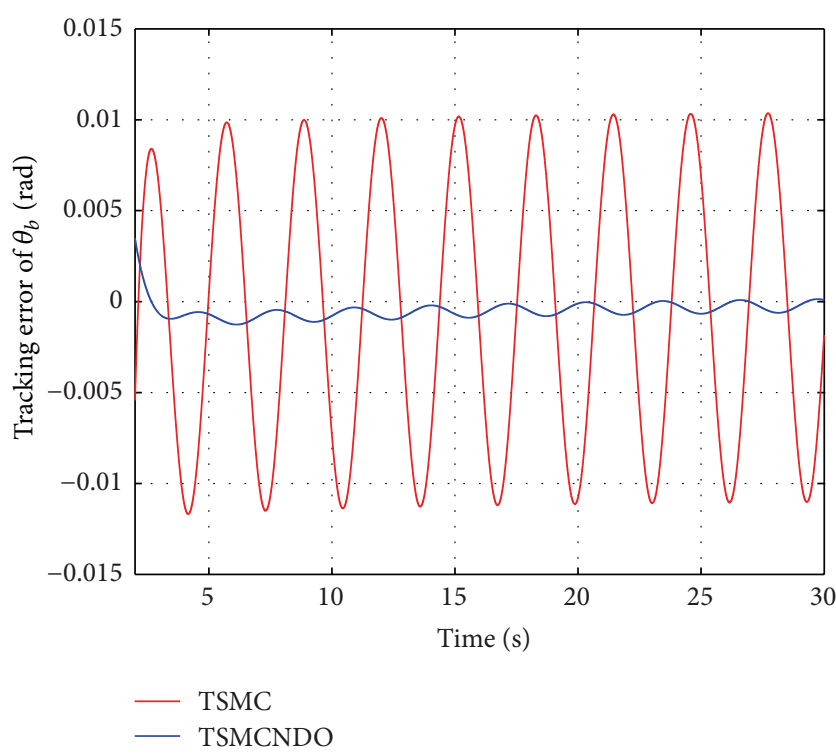

FIGURE 6: Inclination angle tracking errors of the MWIP system by employing TSMC and TSMCNDO control strategies $\left(\theta_{b}(0)=-10^{\circ}\right.$, $\phi_{\text {TSMC }}=\phi_{\text {TSMCNDO }}=0.05$.

large. This is because the NDO can compensate the lumped disturbance in a feedforward way.

In a word, the proposed TSMCNDO control strategy is superior to a conventional LQR or TSMC strategy in the balance control of an MWIP system.

\section{Conclusion}

The balance control of MWIP system is a challenge due to its strong nonlinearity and underactuated feature. The TSMC seems an appropriate method because it can deal with both the modeling uncertainties and external disturbances. In addition, a TSMC controller can guarantee the system trajectory converges in a finite time, whereas there are few researches about the robust finite-time control strategy applied in an underactuated system such as an MWIP. The main contribution of this paper lies in the following.

(1) We formulated the TSMC design for the balance control of the underactuated MWIP system.

(2) To remove the "chattering" caused by sliding mode control and further improve the control performance, a new TSMCNDO strategy is proposed for controlling the MWIP system.

Together with the nonlinear disturbance observer, the control precision is significantly enhanced by the proposed method even if the boundary layer parameter $\phi$ is relatively large. Simulation results demonstrate the effectiveness of the proposed methods.

\section{Conflict of Interests}

The authors declare that there is no conflict of interests regarding the publication of this paper.

\section{Acknowledgments}

This work was partially supported by the International Science and Technology Cooperation Program of Hubei Province "Joint Research on Green Smart Walking Assistance Rehabilitant Robot" under Grant no. 2012IHA00601, the Fundamental Research Funds for the Central Universities (HUST) under Grant no. 2013ZZGH007, and the National Natural Science Foundation of China under Grant 61473130 and was partially supported by Program for New Century Excellent Talents in University (Grant no. NCET-12-0214).

\section{References}

[1] D. Kamen, "Segway Company," San Francisco, Calif, USA, 2010, http://www.segway.com.

[2] F. Grasser, A. D’Arrigo, S. Colombi, and A. C. Rufer, "JOE: a mobile, inverted pendulum," IEEE Transactions on Industrial Electronics, vol. 49, no. 1, pp. 107-114, 2002.

[3] J. Huang, F. Ding, T. Fukuda, and T. Matsuno, "Modeling and velocity control for a novel narrow vehicle based on mobile wheeled inverted pendulum," IEEE Transactions on Control Systems Technology, vol. 21, no. 5, pp. 1607-1617, 2013.

[4] M. Sasaki, N. Yanagihara, O. Matsumoto, and K. Komoriya, "Steering control of the Personal riding-type wheeled Mobile Platform (PMP)," in Proceedings of the IEEE IRS/RSJ International Conference on Intelligent Robots and Systems (IROS '05), pp. 1697-1702, August 2005.

[5] A. Salerno and J. Angeles, "The control of semi-autonomous two-wheeled robots undergoing large payload-variations," in Proceedings of the IEEE International Conference on Robotics and Automation, pp. 1740-1745, May 2004.

[6] K. Pathak, J. Franch, and S. K. Agrawal, "Velocity and position control of a wheeled inverted pendulum by partial feedback linearization," IEEE Transactions on Robotics, vol. 21, no. 3, pp. 505-513, 2005.

[7] C. Li, X. Gao, Q. Huan et al., "A coaxial couple wheeled robot with T-S fuzzy equilibrium control," Industrial Robot, vol. 38, no. 3, pp. 292-300, 2011.

[8] S. Jung and S. S. Kim, "Control experiment of a wheeldriven mobile inverted pendulum using neural network," IEEE Transactions on Control Systems Technology, vol. 16, no. 2, pp. 297-303, 2008.

[9] Z. Li, C. Yang, and L. Fan, Advanced Control of Wheeled Inverted Pendulum Systems, Springer, London, UK, 2013.

[10] V. Sankaranarayanan and A. D. Mahindrakar, "Control of a class of underactuated mechanical systems using sliding modes," IEEE Transactions on Robotics, vol. 25, no. 2, pp. 459-467, 2009.

[11] B. S. Park, S. J. Yoo, J. B. Park, and Y. H. Choi, "Adaptive neural sliding mode control of nonholonomic wheeled mobile robots with model uncertainty," IEEE Transactions on Control Systems Technology, vol. 17, no. 1, pp. 207-214, 2009.

[12] J. Huang, Z.-H. Guan, T. Matsuno, T. Fukuda, and K. Sekiyama, "Sliding-mode velocity control of mobile-wheeled invertedpendulum systems," IEEE Transactions on Robotics, vol. 26, no. 4, pp. 750-758, 2010.

[13] M. Zhihong, A. P. Paplinski, and H. R. Wu, "A robust MIMO terminal sliding mode control scheme for rigid robotic manipulators," IEEE Transactions on Automatic Control, vol. 39, no. 12, pp. 2464-2469, 1994. 
[14] K.-B. Park and T. Tsuji, "Terminal sliding mode control of second-order nonlinear uncertain systems," International Journal of Robust and Nonlinear Control, vol. 9, no. 11, pp. 769-780, 1999.

[15] H. Bayramoglu and H. Komurcugil, "Nonsingular decoupled terminal sliding-mode control for a class of fourth-order nonlinear systems," Communications in Nonlinear Science and Numerical Simulation, vol. 18, no. 9, pp. 2527-2539, 2013.

[16] H. Bayramoglu and H. Komurcugil, "Time-varying slidingcoefficient-based terminal sliding mode control methods for a class of fourth-order nonlinear systems," Nonlinear Dynamics, vol. 73, no. 3, pp. 1645-1657, 2013.

[17] A. Mohammadi, M. Tavakoli, H. J. Marquez, and F. Hashemzadeh, "Nonlinear disturbance observer design for robotic manipulators," Control Engineering Practice, vol. 21, no. 3, pp. 253-267, 2013.

[18] W.-H. Chen, "Disturbance observer based control for nonlinear systems," IEEE/ASME Transactions on Mechatronics, vol. 9, no. 4, pp. 706-710, 2004.

[19] J. Wu, J. Huang, Y. Wang, and K. Xing, "Nonlinear disturbance observer-based dynamic surface control for trajectory tracking of pneumatic muscle system," IEEE Transactions on Control Systems Technology, vol. 22, no. 2, pp. 440-455, 2014.

[20] X. Wei, H. Zhang, and L. Guo, "Composite disturbanceobserver-based control and terminal sliding mode control for uncertain structural systems," International Journal of Systems Science, vol. 40, no. 10, pp. 1009-1017, 2009.

[21] J. Yang, S. Li, and X. Yu, "Sliding-mode control for systems with mismatched uncertainties via a disturbance observer," IEEE Transactions on Industrial Electronics, vol. 60, no. 1, pp. 160-169, 2013. 


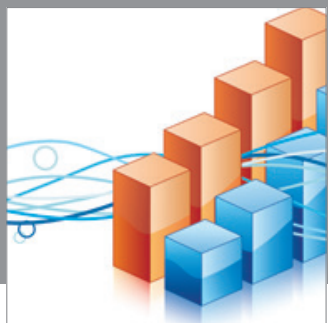

Advances in

Operations Research

mansans

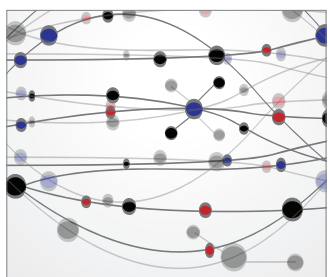

The Scientific World Journal
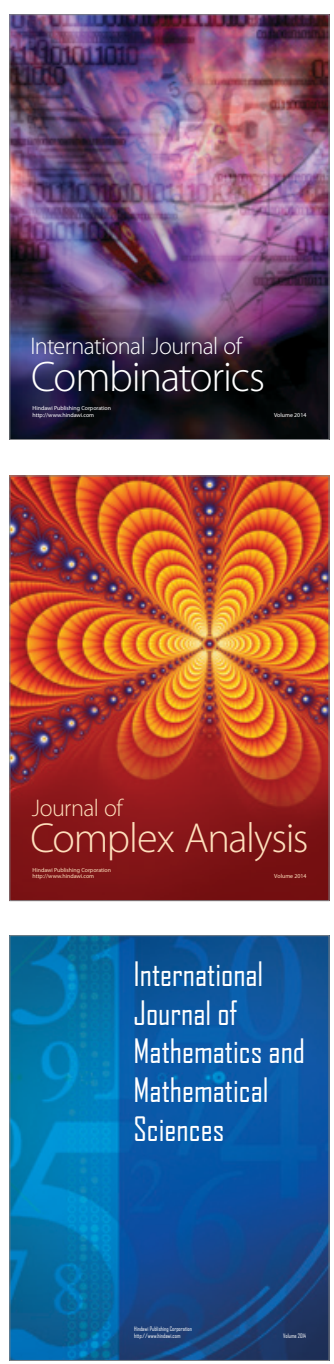
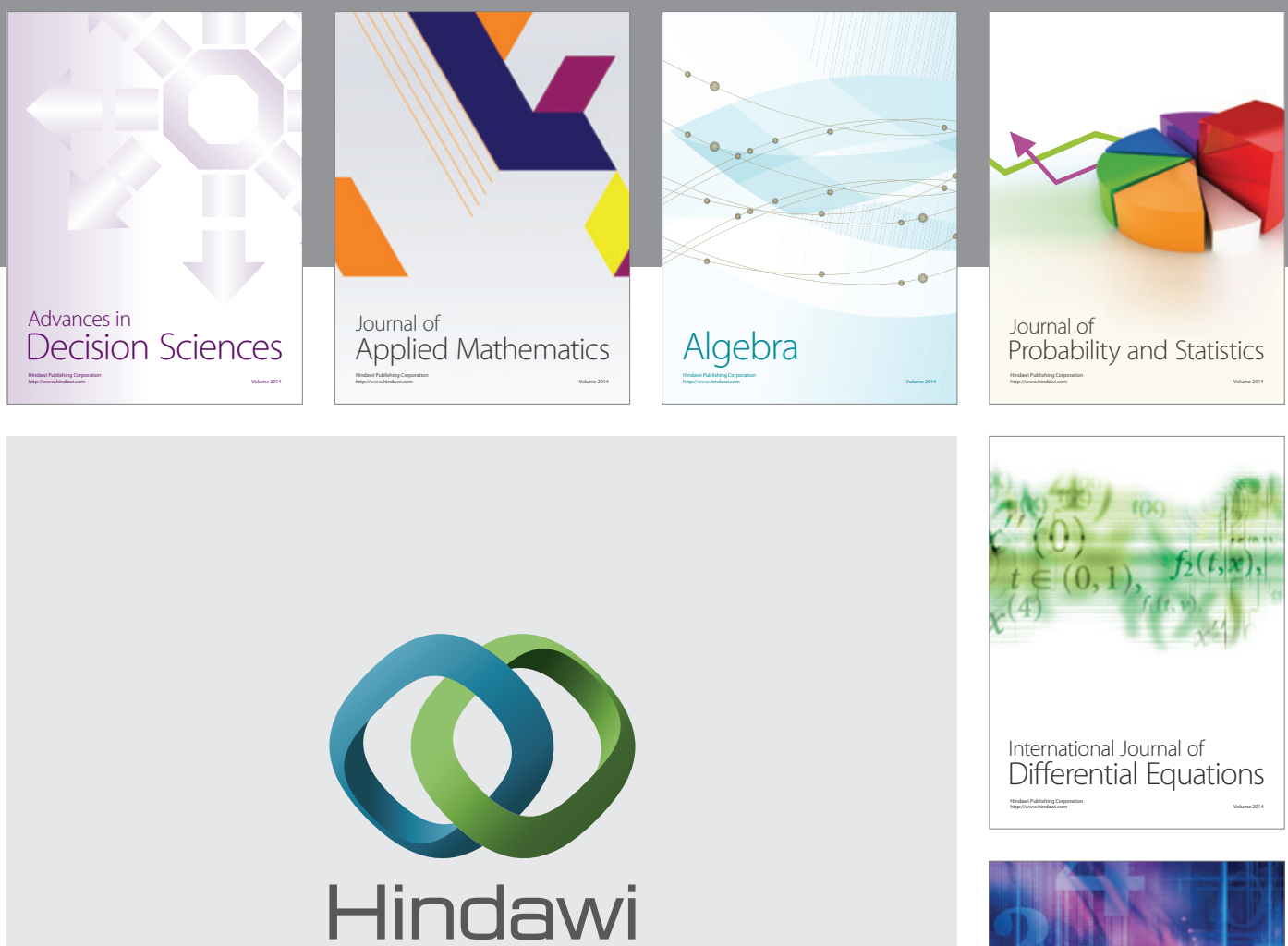

Submit your manuscripts at http://www.hindawi.com
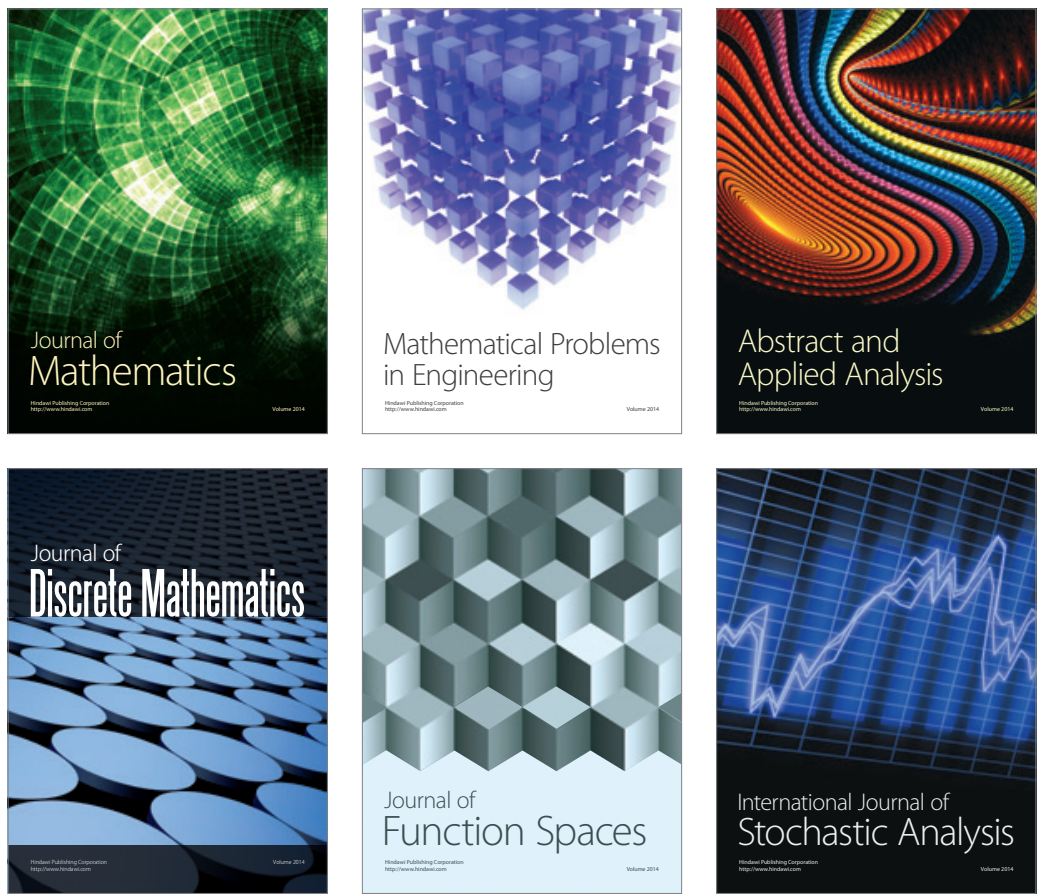

Journal of

Function Spaces

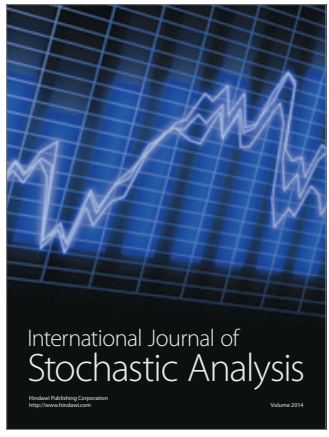

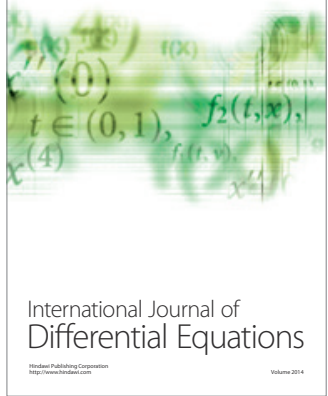
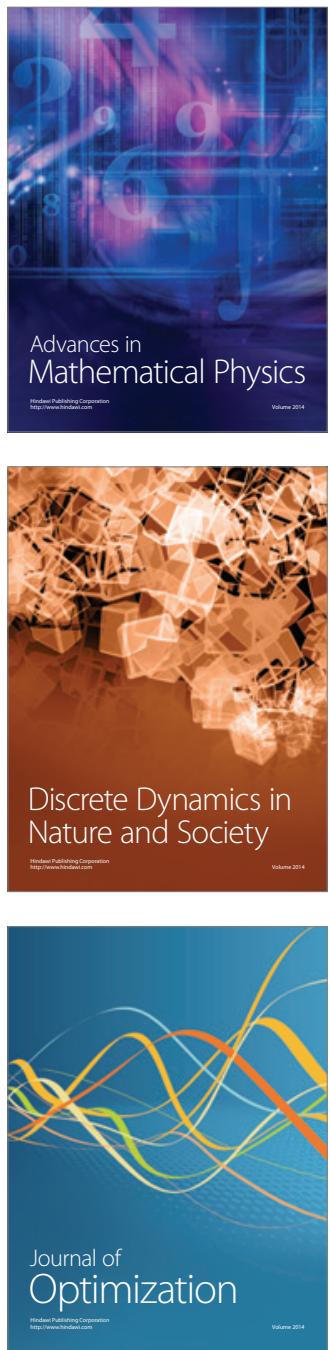